January 2018, Volume 2, Issue 1

\title{
A Survey on Random Walk-Based Stochastic Modeling in Eukaryotic Cell Migration with Emphasis on its Application in Cancer
}

\author{
Fateme Safaeifard $^{1}$, Seyed Peyman Shariatpanahi ${ }^{1, *}$, Bahram Goliaei ${ }^{1}$
}

\author{
${ }^{1}$ Institute of Biochemistry and Biophysics, University of Tehran, Tehran, Iran \\ * Corresponding author: Seyed Peyman Shariatpanahi, Institute of Biochemis- \\ try and Biophysics, University of Tehran, Tehran, Iran,
}

DOI: 10.30699/acadpub.mci.2.1.1Ｅmail: pshariatpanahi@ut.ac.ir

Submitted: 5 July 2017

Revised: 17 September 2017

Accepted: 10 October 2017

ePublished: 01 January 2018

\section{Keywords:}

Cell Migration

Random Walk

Cancer

\begin{abstract}
Impairments in cell migration processes may cause various diseases, among which cancer cell metastasis, tumor angiogenesis, and the disability of immune cells to infiltrate into tumors are prominent ones. Mathematical modeling has been widely used to analyze the cell migration process. Cell migration is a complicated process and requires statistical methods such as random walk for proper analysis. In this study, we reviewed the studies conducted on the random walk-based stochastic modeling of the eukaryotic cell migration. This statistical modeling can open some perspectives on more detailed modeling approaches and ultimately lead to more comprehensive knowledge about the biophysical and biochemical foundations of cell migration process.
\end{abstract}

(C) 2018. Multidisciplinary Cancer Investigation

\section{INTRODUCTION}

The Eukaryotic cell migration, a complicated process, which is highly regulated by molecular pathways, plays a significant role in organogenesis and tissue development. Most cells demonstrate oriented or random migration in vitro, although they may have been extracted from tissue composed of immobile cells [1]. Cell migration disorders in developed organisms are mostly associated with impairments, such as cancer cell metastasis, immune diso-rders, and wound healing deficiency. Particularly in cancer, cell migration depends on the incidences of specific mutations [2] where extensive movement occurs in metastatic cells [3]. In addition, some studies have indi-cated that patients developing tumors with the elevated levels of tumor-infiltrating cytotoxic T lymphocytes have improved response to the treatment. In this regard, one of the main goals of cancer immunotherapy is to control tumor-immune system interactions [4].

The leading studies on cell migration process are focused on fibroblasts, keratinocytes, and myoblasts. Despite some differences in the migration process of various eukaryotic cells, studies have revealed basic similarities in the mechanisms involved in morphogenesis, wound healing, immune response, and cancer cell metastasis [5]. The quantification of these similarities and differences helps in categorizing and modeling of the process. These models predict the migration properties and help in understanding the speed of cell motion-dependent processes, such as metastasis, the characteristics of an optimum migration behavior for effective interaction between lymphocytes and infectious cells and tumor, and the migration ability of tumor cells considering the spatial heterogeneity of tumor.

At present, mathematical modeling and numerical simulations are abundantly applied as complementary to in vitro investigations. Despite the perpetual necessity of experimental approaches for understanding biological processes, in silico experiments can complement these assays by limiting the measuring space and reducing the experiment cost [6-8].

The main advantage of in silico computational models is the simultaneous analysis of several dependent variables that presently seems unatt-ainable experimentally. The evaluation of the relationships of systems' variables, in spite of non-linear dynamics, facilitates more compreh-ensive and accurate analyses of experimental observations. In addition, mathematical models can identify key parameters, determining the ultimate behavior of the system and suggest the most precise experiments for system assessment [9].

The processes associated with cell migration are in- 
fluenced by several closely related factors such as extracellular matrix, tissue architecture, and different cell characteristics. These factors determine the ultimate mode of cell movement in an integrative manner [10]. The time-lapse microscopy of living cells, followed by the applicat-ion of image processing algorithms, conventionally introduces a typical cell migration study. The image processing procedure is initiated with the application of image preprocessing algorithms to enhance signal-to-noise ratio and improve image quality. The application of the algorithms generally depends on the type of applied microscopy technique. The next step is to track the cell migration path and quantitatively estimate cell motility behavior using image processing software and tools [11]. The methods may include particle tracking techniques relied on image segmentation algorithms $[12,13]$, fitting particle path curve to an evolving model, and minimizing the relevant energy functions [14], as well as the estimation of cell prospect using the posterior density function of cell states derived from Monte Carlo sampling methods [15]. The procedure terminates by recording cell spatial coordinates over the time and quantifying cell motion based on reconstructed trajectories. Accordingly, a set of parameters and quantities such as speed, rotation angle, and distance traveled can be derived for inferential data analysis [11]. At present, various software tools are available for digital image processing where some of them implement algorithms adapted for the analysis of migrating cells.

Cell movement is a stochastic process and the derivation of its descriptive parameters requires a clear discrimination of stochastic patterns relied on the rules governing random models, such as random walk. This field of applied mathematics is increasingly used to model biological systems, in particular in ecology (animals' movement) and pathophysiology (cell movements, particularly to form blood vessels and metastasis). The main advantage of random walk models is its ability to differentiate migration characteristics through intuitive data by which different cell migration mechanisms can be better understood [16].

The time series analysis of cell trajectories provides quantitative data for the reconstruction of cell-specific motility models. Such macroscopic models of cell behavior with the microscopic properties of cellular dynamics, such as cytoskeletal changes, cell contraction forces, and the dynamics of adhesion molecules, define the systems biology of cell motility [17].
The Simple Random Walk Model (Diffusion Equation)

The Brownian motion is the foremost example of a random process (Figure 1a), which follows the simple random walk rules. In the simplest case, a particle in one-dimensional space can move from the origin with the same probability of left to right and right to left motions, where its displacement, $\Delta \mathrm{x}$, is constant in constant discrete time intervals. In this model, particle movement does not depend on its previous and current positions, as well as on time; thus, the average displacement after time $\mathrm{T}$ is zero. Hence, to define a significant variable for the amount of displacement, the mean squared displacement (MSD) of the particle after a walk of $\mathrm{N}$ steps is a more suitable statistical quantity [18]. The quantity is calculated using the following formula:

$$
R^{2}(\tau)=\lim _{t \rightarrow \infty}\left\langle(X(t+\tau)-X(t))^{2}\right\rangle
$$

where $|\mathrm{X}(\mathrm{t}+\tau)-\mathrm{X}(\mathrm{t})|$ is the particle displacement between two steps in the time series, $t$ is the total time of the walk, and $\tau$ is the time interval between the successive steps [19]. MSD indicates the average walker distance from the original position as a function of $t$ [20].

For a simple random walker model, we have $\mathrm{R}^{2}(\tau)=2 \mathrm{D}$ $\mathrm{n} \tau$, where $\mathrm{D}$ is the diffusion constant and $\mathrm{n}$ is the spatial dimension [18]. Noting that the evaluation of statistical variables in a complex set of random walkers leads to the spatiotemporal continuity of non-random variables. Such continuous processes are expressed by diffusion models derived from Brownian motion [21]. Specifically, Einstein in 1905 demonstrated that the random motion of particles at microscopic levels can perfectly express the inter-relationship between macroscopic parameters, such as diffusion coefficient, viscosity, and temperature [22,23].

The simple random walk model has repeatedly been used as one of the simplest random models of cell migration. Although this model provides a naive expression for cell migration, it is satisfactory in some cases to express cell migration aspects and diffusion processes using the pure random walk model. For example, the organization of human mesenchymal stem cells on nanotopographical surfaces can be simulated on the basis of a simple random walk model. The results of such stimulations predict the regular and irregular cellular organizations for low and high cellular densities, respectively [24].

\section{Generalization of Simple Random Walk Model}

In most cases, the movement of individual cells is not attributed to the simple random walk behavior. In 
such cases, the evaluation of MSD function results in a more generalized equation, which is as follows:

$$
R^{2}(\tau)=\lim _{t \rightarrow \infty}\left\langle(X(t+\tau)-X(t))^{2}\right\rangle \propto \tau^{\alpha}
$$

In this generalized model, the diffusion phenomenon is categorized on the basis of the parameter $\alpha$. When MSD is the linear function of time $(\alpha=1)$, we obtain the normal diffusion characteristic. If $\alpha>1$, the process is called super-diffusion (rapid diffusion), whereas $\alpha<1$ represents sub-diffusion (slow diffusion) phenomena [25].

In addition to MSD, velocity autocorrelation function is studied as another statistical average to investigate the random walk characteristics as defined elsewhere [26]:

$$
C_{V}(\tau)=\lim _{t \rightarrow \infty} \frac{\langle V(t+\tau) V(t)\rangle}{\left\langle V^{2}(t)\right\rangle}
$$

This function indicates the correlation between the velocity time series of a particle with its time-shifted version. The function is expected to exponentially decrease by the amount of time shift interval $(\tau)$. Regarding cell migration, the function indicates how long the cell moves in a certain direction without a significant change in velocity. For the simple random walk model, the function has non-zero value only when $\tau=0$, as the successive steps in this model are independent [20].

In addition, the probability distribution and density functions indicate the possible values of discrete and continuous random variables, respectively. The probability density functions of variables, such as velocity, step length, and angular displacement, are some other statistical functions, and their behaviors can be interpreted as different characteristics for the random walk model of cell motilities. In the pure random walk model, it is presumed that the distribution of step length and consequently cell speed follow a normal (Gaussian) distribution or take a constant value, whereas cell migration does not confirm the simple assumption in several cases.

\section{Random Walks with Non-Gaussian Step Length Distributions}

Walker step length can follow the desired distribution function. New assumptions for the distribution function of random walk steps can result in super-diffusion or sub-diffusion phenomena, irrespective of steps correlation. In contrast to the classic random walk with a normal distribution with finite variance, Levy walk has Levy flight distribution function with infinite variance. The distribution function reduces with the power law $\mathrm{f}_{\mu}(1) \sim 1^{-\mu}(1<\mu<3)$, where 1 is the step size. Evidently, although long steps are significantly less than short ones, they are relatively much more frequent than the normal distribution (Figure 1b). Levy walk is considered a scale-free random walk, as the power law distribution of step length shows similar behaviors in different spatial scales [27]. In addition, the scale-free nature provides special functionalities as a characteristic of several biological phenomena, such as fractal pattern in human blood vessels [28].

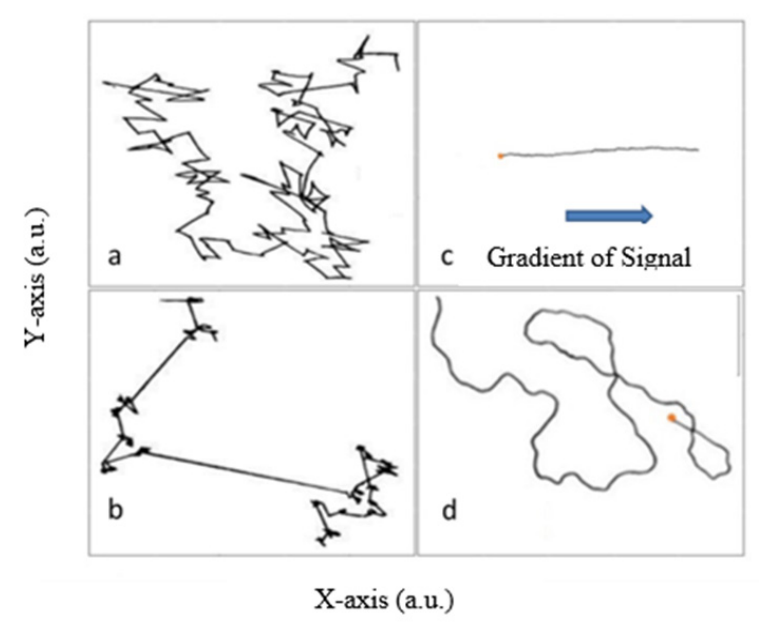

Figure 1: Schematic View of Walker Trajectories in (a) a Pure Random Walk, (b) Levy Walk, (c) Biased Random Walk, and (d) Persistent Random Walk.

It was suggested that lymphocytes motility into the brain does not follow Brownian statistics but can be described satisfactorily on the basis of the generalization of Levy walks. The model captures run and pause phases observed in lymphocytes motility. Such migration enables cells to identify rare far goals with more than 10-fold efficiency; compared to Brownian motion, this migration strategy is similar to that of monkeys and marine predacious [29].

In addition, the motility of hydra cells is super-diffusion, with non-Gaussian velocity distribution in both ectodermal and endodermal tissues. The diffusion of hydra cells can be explained as a correlated type of anomalous diffusion using Tsallis statistics [30,31]. In addition to Gaussian and Levy distributions, other statistical distributions have also been used to express the specificity of cell migrations; for instance, the migration of normal and tumor melanocyte cells in mice demonstrates a transition from a normal diffusion $(\alpha=1)$ to super-diffusion $(\alpha>1)$ without a long-time angular persistence. The non-Gaussian velocity distribution of the cells introduces a 
q-Gaussian model of migration and simulation of cell trajectories. The model perfectly reproduces the displayed transition phenomenon [17]. Moreover, the migration study of normal and tumor epithelial cells derived from Madin-Darby canine kidney (MDCK) cell line revealed the diffusion anomaly of the cells. The evaluation of the cell speeds indicated that velocity proportions generated a q-Weibull distribution, an extension of the Weibull distribution accounted for Tsallis distribution function:

$$
\mathrm{p}_{\mathrm{qw}}(\mathrm{x})=\mathrm{p}_{0} \frac{\mathrm{rx}^{\mathrm{r}-1}}{\mathrm{x}_{0}^{\mathrm{r}}} \exp _{\mathrm{q}}\left[-\left(\frac{\mathrm{x}}{\mathrm{x}_{0}}\right)^{\mathrm{r}}\right]
$$

where the q-exponential expression $\left(\exp _{\mathrm{q}}(\mathrm{x})\right)$ equals to $[1+(1-q) x]^{\frac{1}{1-q}}$. Podesta et al. showed that the cell density variations result in the alternation of the shape parameters of the distribution ( $\mathrm{r}$ and $\mathrm{q}$ ) in a way that the distribution function inclines to Maxwellian distribution as the density of the cells increases [32]. Biased Random Walk Model

The directionality of locomotion in response to factors, such as the mechanical properties of the environment and the concentration of particular chemical compounds, is one of the vital characteristics of some eukaryotic cells [33-35]. Such migration is characterized on the basis of the non-uniform distribution of steps orientation [27]. Chemotactic movement, the most stated example of this migration pattern, can be found in specific types of eukaryotic cells (Figure 1c) [33,36-38]. For instance, experimental assessments indicated that the chemo-taxis of neutrophils in three-dimensional culture systems supported the predictions of the biased random walk (BRW) model for this migratory behavior [39]. The evaluation of B-cells> chemotaxis revealed that the highest chemotactic responses correspond to the medium gradient of chemokines, and thus, the autocorrelation function showed slower decrease than gentle and rigorous gradients [40].

In addition, spatial constraints affect the coordinated According to the average dynamics of single cells in the colonies of HeLa cells and its dependence on spatiotemporal heterogeneity associated with growth geometry, Muzzio et al. suggested that cell mobility was achieved by a concerted mechanism. In this mechanism, two limiting elements with a random walk and ballistic motion $(\alpha>1)$ participate to form a BRW migration. The directional biases in single cell motion influence the dynamics of the two-dimensional colony development and their specific characteristics [44].
Furthermore, the experimental findings revealed a migration tendency of cells toward the stiffness gradient of the extracellular matrix. However, different assays confirmed the increased directional persistence of migrating cells with increasing matrix stiffness $[45,46]$. On performing random walk-based simulations, Novikova et al. uncovered that the net flow of the cells is the conclusive outcome of the persistence subordination of matrix stiffness [47].

\section{Persistent Random Walk}

In the pure random walk model, step orientation, as well as turning angles, is considered to be independent of the trajectory in each step. In more realistic models, the natural attitude of the cell to preserve the last step's orientation is specified by the non-uniform distribution functions of turning angles [27]. Signaling pathways lead to the formation or destruction of cytoplasmic appendages (lamellipodia and filopodia) in the cell migration process mainly by the activation or deactivation of factors associated with the formation of actin filaments organization in the different areas of the cell membrane. The local activity of protein network involved in cell motility discovers the mechanism of the directional persistency of the cell migration through responding to local mechanical (through cell matrix adhesions) and chemical signals, as well as branching new appendages of the older ones. This branching proposes a positive feedback mechanism by which migration occurs persistently in the absence of any external signal (48-50). As assumed in the persistent random walk (PRW; also called correlated random walk), the cell persists in its direction of motion in some timescales, whereas displays a type of random movement along its trajectory in longer time scales (Figure 1d) [51]. Consequently, in the MSD diagrams, a continuous change from a more ballistic behavior $(\alpha>1)$ for shorter time intervals to Brownian motion pattern $(\alpha=1)$ for longer time distance is observed [52,53].

Notably, because of this discrepancy in different time lags, in cell migration studies, sampling time intervals may influence the results of statistical analyses of the obtained time series. Rosser et al. (2013) used a stochastic model to study the distribution of velocity and turning angles found from different sampling intervals. The results showed that changing time intervals considerably changes the above quantities up to several orders of magnitude [54].

The motion of several individual cells can be evaluated on the basis of the PRW model. In this regard, several efforts were made to model the involved mechanisms to elucidate the dependence of two consecutive steps in a random motion. Several models 
cover the cell membrane fluctuations as a stochastic factor and actin network orientation as a persistence factor. For instance, Caballero et al. showed that the direction of cell motion is predictable using a statistical analysis derived from the dynamics of cytoplasmic appendages. The developed model indicated that asymmetry in the fluctuations of membrane protrusions was sufficient to predict the data obtained from the motion called biased PRW [55].

Furthermore, a model was developed by Cooper et al. (2012) on the basis of cell excitability and plasma membrane retention to describe the mechanism of the directional persistence of the cells employing a zigzag model of the consecutive formation of the protrusion. Considering the spatial limitation of cell membrane after pseudopod formation, as well as the excitation memory in the area of older pseudopod, their model could reproduce experimental data on cell migration. In addition, comparison of the predictions of this model with the experimental data indicated that the pseudopod formation was a non-Markovian process influenced by the dynamics of older pseudopodium [56].

Another model was proposed to survey the physical basis of the migration of amoeboid cells, such as Dictyostelium, and the epithelial cells of MDCK as well. In this model, the accumulation of actin polymerization inhibitors in the tail end of the cell acts as the operant of forwarding persistent motion. In addition, the density fluctuations of these molecules along with membrane drops and rises are considered as the random factors, influencing the directionality and reorientation of the cell. The combination of the stochastic turns and directional motions resulted in the anomalous diffusion (super-diffusion) of the cells in signal-absent conditions [57,58]. Furthermore, the analysis of the probability distribution of the angles between pseudopods illustrates another method to develop a stochastic model for the mechanism of cell mobility inspired from the correlated random walk. For example, a Monte Carlo simulation is proposed that incorporates the proportion of the number of pseudopods bifurcating from the older ones to the number of randomly formed de novo protrusions, pseudopodium's directional alteration (left to right and vice versa), their angular separation, and the angle variance [59].

Moreover, it is shown that cellular persistence is an exponential function of speed; this general rule can be explained on the basis of a physical model of cell polarity changes along with actin network dynamics [60] and its effect on cell directional persistency. This model can predict a general phase diagram of cell trajectories, which reproduces a full range of observed migration patterns [61].

In some cell populations, a combination of simple random walk and PRW models can be used to explain cell migration properties; for example, lymphocytes migration is predictable considering such two-population models. However, the application of PRW model for exploring motile lymphocytes in lymph nodes, in general, had been confirmed experimentally $[62,63]$, It was shown that $\mathrm{CD}^{8+} \mathrm{T}$-cells migration in non-inflamed lymph nodes does not completely comply with the PRW model. In turn, the two-population model, suggested by Banigan et al., attributes Brownian motion and variable PRW model with noise to different cell populations, and can predict the experimental statistical properties of the migration of such cells. The discrepancy between two-population model and the model proposed to describe active T-cells migration inside inflammatory brain demonstrates the discordance of statistical features related to lymphocytes migration in different tissues [29,64].

In addition, in a study on the motility of B lymphocytes in germinal centers, O'Conner et al. showed that unlike the random motion of inactive B-cells, active B-cells show biased motility and the slope of their MSD plot accepts an $\alpha$-value of more than 1 [65]; this is similar to the behavior of primary blood cells, which follow the PRW migration model to reach medullary cords [66].

The capability of direction retention, inducing persistent random motion, results in some complicated behaviors in several cells. The migration behavior of an amoeboid eukaryotic cell was investigated in the absence of chemotaxis signals, and the results indicated that the cell displayed a particular model of random motion under following circumstances: long intervals of straight motion and frontward movement with zigzag steps. The motion comprises the consecutive phases of run and turn and the migrating cells have a directional memory of one step. The distribution of traveled distance and turning magnitude can be fit to an exponential function. This migration model can increase the chance of acquiring targets, compared with Brownian motion [52,67].

The physical properties of the environment influence the migration of individual cells (68). Regarding the migration of hydra cells in ectodermal and endodermal communities, Rieu et al. (2000) applied the standard statistical analyses and accordingly showed that the cell motion can be explained in both structures on the basis of the PRW model. Different diffusion coefficients were found for the two communities, 
where the smaller diffusion coefficient for the endoderm indicated its more adherent structure [69].

In addition, the effect of interstitial flow on cell migration demonstrated that increased flow changes breast tumor cells to an amoeboid phenotype. The evaluation of the heterogeneity of migratory phenotypes of cells subjects to different interstitial flow intensity and ascertains other random walk characteristics for amoeboid and mesenchymal phenotypes, among them was distinct directional persistence in two mentioned migration modes [70,71].

\section{GENERALIZED PERSISTENT RANDOM WALK}

\section{Persistent Random Walk Models with Non-Gauss- ian Step Length Distribution}

In this generalization, the assumption of the constant or Gaussian step length has been reduced; hence, the distribution of steps length may follow an arbitrary function.

For instance, by the evaluation of the migration of human breast epithelial cells, the bimodal correlated random walk model as an alternative to the simple PRW model was proposed. This model of movement comprises two types of correlated flights with exponential distribution: a first directional phase that can be replaced by a second reorientation phase successively [6].

Additionally, while investigating the motion of $\mathrm{T}$ lymphocytes in lymph nodules, Fricke et al. reported that likewise Brownian motion, Levy walk is not a full descriptive model of migration. As an alternative, they suggested a correlated random walk with the peculiar distribution function of step length. The lognormal distribution function of velocities, as well as steps length, short-term directional persistence, and heterogeneity of cell mobility patterns, are main properties that enhance the proficiency of this migration pattern. The evaluation of the average velocity of studied $\mathrm{T}$ lymphocytes revealed that $\mathrm{T}$-cells show a continuous pattern of motion, which results in the defined search strategies: cells have a relatively smaller average velocity with a heavy-tailed distribution that permits them to have few long-range steps. The velocity distribution of mentioned cells shows the most compliance with lognormal distribution with parameters $\mu$ and $\sigma$ that equal to 0.48 and 0.91 , respectively:

$$
\begin{gathered}
\mathrm{y}=\mathrm{f}(\mathrm{x} \mid \mu, \sigma)=\frac{1}{\mathrm{x} \sigma \sqrt{2 \pi}} \mathrm{e}^{\frac{-(\ln \mathrm{x}-\mu)^{2}}{2 \sigma^{2}}} \\
\sigma=0.91, \mu=0.48
\end{gathered}
$$

The significant characteristic of the distribution is its high measures of skewness and kurtosis in contrast to the fast cells that indicate a more Gaussian-like distribution with lower amounts of skewness and kurtosis. In addition, agent-based simulations depicted that the lognormal modulated PRW has the maximum compliance with the observed efficiency of migrating $\mathrm{T}$ cells, following dendritic cells. This migration behavior presents a balance between the accuracy and amount of distance traveled by the cells [72]. Moreover, the findings confirm the hypothesis of migration heterogeneity among the T-lymphocyte population [64].

\section{Persistent Random Walk of Cells as Heteroge- neous Time Series}

In some cases, the temporal homogeneity of cell migration properties should be considered by the models. For instance, epithelial cells may find the movement potential through a process called epithelial to mesenchymal transition that occurs in cancer cells metastasis $[73,74]$. To model cell migration based on time-dependent quantitative properties, one of the possible approaches is the estimation of statistical variables through time; Bayesian inference, developed in the shadow of Bayesian statistics, can be used for this propose. The Bayes theorem relates the probability distribution of a random variable without any knowledge of observations (prior distribution) to its probability distribution after the observation of the event (posterior distribution). Applying Bayesian inference method, based on Bayes theorem, the statistical properties of random variables can be updated after an initial estimate of their distribution [75]. Metzner et al., resembling the cell migration phenomenon as a random walk with time-varying statistical parameters, showed that the time dependent parameters can be estimated in consecutive steps of obtained time series using the sequential Bayesian inference method. The parameters of the migration model studied were cellular activity and persistence that showed considerable fluctuations through investigated cell trajectories. The possibility of evaluating the effect of environmental characteristics on cells motility is another result obtained using sequential Bayesian inference method [76].

In addition, it has been shown that a Bayesian inference schema can be established to evaluate the accordance between a class of random walk and the observed cell trajectories' data. Within this framework, the migration patterns of zebrafish macrophages and neutrophils showed distinct random walk models whose amounts of directional persistence were spatiotemporally modulated [77]. 


\section{Anisotropic Persistent Random Walk}

The PRW model cannot be considered as the best description for the motility profile of all cell species. A more comprehensive model can be developed considering generalizations, such as the heterogeneity of migration behaviors among cell population, as well as excluding the assumption of spatial isotropy in velocities of the cells. The migration pattern of fibrosarcoma cells in two-dimensional and three-dimensional cultures showed some deviations from the PRW model, among which is the non-Gaussian distribution of speeds that emerge from the heterogeneous nature of cell migration. Wu et al. suggested that two-dimensional migration statistics can be well predicted by the PRW model considering different model parameters for individual cells. However, the simulation results showed that predicting three-dimensional cell migration characteristics, additionally, requires the attribution of different persistent times and speeds to the primary and non-primary directions of movement. The anisotropic PRW model perfectly predicts the migration behavior of the cells. [78].

\section{Reinforced Random Walk}

In this model, it is assumed that the walker moves on a weighted graph. The probability of choosing the edges connected to the vertex is proportional to the weights attributed to them. In addition, after each walk through an edge, the weight may increase [79]. This weight amplification can be interpreted as the creation of a cell pathway in the extracellular matrix in such a way that the probability of cell migration in the certain direction increases. Similarly, adding the appropriate initial weight to the edges of the graph, a phenomenon such as chemotaxis can also be modeled on the basis of this type of random walk $[80,81]$. Angiogenesis is one of the physiological processes illustrated by this model. A mathematical model based on the theory of reinforced random walk developed by Plank et al., (2002) successfully described the migration of vascular endothelial cells in response to different angiogenesis factors. According to this model, endothelial cells initially form a spherical community in which all cells can move on a regular graph. Chemotaxis responding to vascular endothelial growth factor (VGEF) and haptotaxis responding to collagen concentration are modeled by adding proper weights to the edges of the graph, proportional to the concentration of the components [82]. Moreover, hybrid models proposed to map VEGF-depended angiogenesis consider that VEGF concentration changes in addition to modeling che- motaxis of endothelial cells using the reinforced random walk. For example, it is reported that the hybrid model was able to predict the threshold concentration required to stimulate the quiet cells for a biased motion, as well as the optimum concentration for their movement [83].

\section{Stochastic Langevin Equations}

The application of stochastic differential equations is another approach to describe the stochastic properties of cell migration phenomenon. The stochastic Langevin equation characterizes the walker movement in a viscous environment under the influence of stochastic forces. The Ornstein-Uhlenbeck $(\mathrm{O}-\mathrm{U})$ process, as the solution of Langevin equations, is a random process evolved from Brownian motion to model the velocity of particles in the fluid as a continuous time random walker $[84,85]$.

This $\mathrm{O}-\mathrm{U}$ process incorporates two factors influencing particle velocity: the frictional resistance of a particle in the fluid proportional to its velocity and stochastic factors such as thermal noise and the random hitting from the particles around $[20,84]$. The applications of both the continuous and discrete representation of $\mathrm{O}-\mathrm{U}$ process have been investigated in cell movement studies [86-89].

The cell motion equations derived from the Langevin equations for a Brownian particle are expressed as follows:

$$
\begin{gathered}
\frac{d x(t)}{d t}=v(t) \\
\frac{d v(t)}{d t}=-\hat{a} v(t)+\sqrt{a} \hat{\imath}(t)
\end{gathered}
$$

where $\beta$ and $\alpha$ express the intensity of viscous and random forces, respectively, and $\xi(\mathrm{t})$ is a random term with specified distribution. By adding an expression to the second equation, the effect of concentration gradient of chemotactic molecules can also be included. The statistical analysis of the process indicates that the two involving parameters, $\alpha$ and $\beta$, are in association with two cell motility parameters, namely, cell speed and persistence time $[87,88]$.

A dynamic model proposed by Liang et al. (2011) on the basis of the experimental data from Dictyostelium cell migration could successfully depict the statistical characteristics obtained from cell trajectories. In this model, using the $\mathrm{O}-\mathrm{U}$ process, two stochastic Langevin differential equations were recommended 
to model the random alternative emergence of pseudopods, perpendicular and parallel with the direction of motion [90].

However, there are some inconsistencies between $\mathrm{O}-\mathrm{U}$ process predictions and the mobility behavior of some cells, such as fibroblasts and keratinocytes. Eventually, cell specificity can be explained by attributing cell-specific values to the parameters included in the model. The specificity of the models indicates that the different migration modes of certain cells can be elucidated by applying relevant models where differences are considered in the values of the model parameters $[91,92]$.

\section{CONCLUSIONS}

Due to the importance of the cell migration process, particularly in the initiation and development of diseases such as cancer, cell migration models have considerably attracted attention in recent years. The mathematical models based on stochastic processes were among the ones that showed their ability in the statistical characterization and classification of cell migration. This study aimed to describe the characteristics and potentials of different types of random walk models regarding the migration of eukaryotic cells. For this purpose, we attempted to classify modeling approaches and introduce the models' application to explain the migration process in different cells. Table 1 briefly shows the different types of the developed models.

In conclusion, studies indicated that the modeling of cell migration on the basis of the mathematical rules in stochastic processes and random walk models revealed some differences and similarities among cell migration strategies in different cell species and under distinct environmental conditions.

\section{ACKNOWLEDGMENTS}

We thank MCI editors for their kind help.

\section{CONFLICT OF INTEREST}

The authors claimed that there is no conflict of interest regarding this paper.

\section{ETHICS APPROVAL}

Not applicable.

Table 1: Random-Walk Models in the Migration of Eukaryote Cells

\begin{tabular}{ll}
\hline \multicolumn{1}{c}{ Random Walk Model } & \multicolumn{1}{c}{ Eukaryote Cells } \\
\hline \multicolumn{1}{c}{ Rimple Random-Walk Model } & \multicolumn{1}{c}{ Inactive B-Cells on Germinal Centers [65] } \\
\hline \multicolumn{1}{c}{ Random Walks with Non-Gaussian Step-Size Distributions } \\
\hline The Q-Gaussian Random-Walk Model & Normal Melanocytes and Melanocytic Tumors in Mice [17] \\
\hline The Q-Weibull Random-Walk Model & Normal Epithelial and Epithelial Tumor Cells [32] \\
\hline Aneralized Levy Walk & Lymphocytes in Brain [29] \\
\hline Persistent Random-Walk Model & Lymphocytes in Lymph Nodes [63] \\
\hline Reinforced Random-Walk Model & Hydra Cells in Cellular Communities [69] \\
\hline Biased Random-Walk Model & Vascular Endothelial Cells [82] \\
\hline Bimodal Correlated Random-Walk Model & Chemotactic Migration of Neutrophils [39] \\
\hline Lognormal Modulated CRW & Human Breast Epithelial Cells [6] \\
\hline Persistent Random-Walk Model of Cell as a Heterogeneous Time Series & Breast Tumor Cells [76] \\
\hline Anisotropic Persistent Random-Walk Model & Tibrosarcoma [78] \\
\hline Stochastic Langevinequations & Dictyostelium [90] \\
\hline
\end{tabular}




\section{REFERENES}

1. Caballero D, Comelles J, Piel M, Voituriez R, Riveline D. Ratchetaxis: Long-Range Directed Cell Migration by Local Cues. Trends Cell Biol. 2015;25(12):815-27. https://doi. org/ 10.1016/j. tcb.2015.10.009 PMID:26615123

2. Romain B, Hachet-Haas M, Rohr S, Brigand C, Galzi JL, Gaub MP et al. Hypoxia differentially regulated CXCR4 and CXCR7 signaling in colon cancer. Mol Cancer. 2014;13(1):58. https://doi.org/10.1186/1476-4598-13-58 PMID:24629239

3. Cai X, Luo J, Yang X, Deng H, Zhang J, Li S et al. In vivo selection for spine-derived highly metastatic lung cancer cells is associated with increased migration, inflammation and decreased adhesion. Oncotarget. 2015;6(26):22905-17. https://doi.org/10.18632/oncotarget.4416 PMID:26090868

4. Oelkrug C, Ramage JM. Enhancement of T cell recruitment and infiltration into tumours. Clin Exp Immunol. 2014;178(1):1-8. https://doi.org/10.1111/cei.12382 PMID: $\underline{24828133}$

5. Friedl P, Wolf K. Tumour-cell invasion and migration: diversity and escape mechanisms. Nat Rev Cancer. 2003;3(5):362-74. https://doi.org/10.1038/nrc1075 PMID: $\underline{12724734}$

6. Potdar AA, Jeon J, Weaver AM, Quaranta V, Cummings PT. Human mammary epithelial cells exhibit a bimodal correlated random walk pattern. PLoS One. 2010;5(3):e9636. https://doi.org/10.1371/journal.pone.0009636 PMID:20224792

7. Le Dévédec SE, Yan K, de Bont H, Ghotra V, Truong H, Danen EH et al. Systems microscopy approaches to understand cancer cell migration and metastasis. Cell Mol Life Sci. 2010;67(19):3219-40. https://doi.org/10.1007/s00018010-0419-2 PMID:20556632

8. Eddy JA, Funk CC, Price ND. Fostering synergy between cell biology and systems biology. Trends Cell Biol. 2015;25(8):440-5. https://doi.org/10.1016/j. tcb.2015.04.005 PMID:26013981

9. Danuser G, Allard J, Mogilner A. Mathematical modeling of eukaryotic cell migration: insights beyond experiments. Annu Rev Cell Dev Biol. 2013;29(July):501-28. https://doi.org/10.1146/annurev-cellbio-101512-122308 PMID:23909278

10. Petrie RJ, Yamada KM. Fibroblasts Lead the Way: A Unified View of 3D Cell Motility. Trends Cell Biol. 2015 Nov;25(11):666-74. https://doi.org/10.1016/j.tcb.2015.07. 013 PMID:26437597

11. Masuzzo P, Van Troys M, Ampe C, Martens L. Taking Aim at Moving Targets in Computational Cell Migration. Trends Cell Biol. 2016;26(2):88-110.https://doi.org/10.1016/j.tcb. 2015.09.003 PMID:26481052

12. Wu Q, Merchant F, Castleman K. Microscope image processing: Academic press; 2010.

13. Sankur B. Survey over image thresholding techniques and quantitative performance evaluation. J Electron Imaging. 2004 Jan;13(1):146. https://doi.org/10.1117/1.1631315.
14. Kass M, Witkin A, Terzopoulos D. Snakes: active contour models. Int J Comput Vis. 1988 Jan;1(4):321-31. https:// doi.org/10.1007/BF00133570.

15. Liu JS. Monte Carlo strategies in scientific computing: Springer Science \& Business Media; 2008.

16. Codling EA, Plank MJ, Benhamou S. Random walk models in biology. 2008;(April):813-34. https://doi.org/10.1098/ rsif.2008.0014.

17. Da Silva PC, Rosembach TV, Santos AA, Rocha MS, Martins ML. Normal and tumoral melanocytes exhibit q-Gaussian random search patterns. PLoS One. 2014;9(9):e104253. https://doi.org/10.1371/journal.pone.0104253 PMID:25203532

18. Nelson P. Biological Physics : Energy, Information, Life. 2002;

19. Despósito MA, Viñales AD. Sub diffusive behavior in a trapping potential: mean square displacement and velocity autocorrelation function. 2009 Feb 16; https://doi.org/ 10.1103/PhysRevE.80.021111

20. Ibe OC. Elements of Random Walk and Diffusion Processes. 2013. 253 pp. https://doi.org/10.1002/9781118618059.

21. Schilling R, Song R, Vondracek Z. Bernstein functions: theory and applications. 2012. https://doi.org/ $\underline{10.1515 / 9783110269338}$.

22. Einstein A. On the Motion of Small Particles Suspended in a Stationary Liquid, as Required by the Molecular Kinetic Theory of Heat. Ann Phys. 1905;322:549-60. https://doi. org/10.1002/ andp.19053220806.

23. Höfling F, Franosch T. Anomalous transport in the crowded world of biological cells. Rep Prog Phys. 2013;76(4):046602. $\quad$ https://doi.org/10.1088/00344885/76/4/046602 PMID:23481518

24. Gallach Pérez D, Punzón Quijorna E, Sanz R, Torres-Costa V, García Ruiz JP, Manso Silván M. Nanotopography enhanced mobility determines mesenchymal stem cell distribution on micropatterned semiconductors bearing nanorough areas. Colloids Surf B Biointerfaces. 2015;126:146-53. https://doi.org/10.1016/jcolsurfb .2014 .11 .047 PMID:25546837

25. Vainstein MH, Lapas LC, Oliveira FA. Anomalous diffusion. arXiv preprint arXiv:08050270. 2008.

26. Despósito MA, Viñales AD. Subdiffusive behavior in a trapping potential: mean square displacement and velocity autocorrelation function. Phys Rev E Stat Nonlin Soft Matter Phys. 2009;80(2 Pt 1):021111. https://doi.org/ 10.1103/ Phys RevE .80.021111 PMID:19792081

27. Benhamou S. How many animals really do the Lévy walk? Ecology. 2007;88(8):1962-9. https://doi.org/10.1890/061769.1 PMID: 17824427

28. Kedzia A, Rybaczuk M, Andrzejak R. Fractal dimensions of human brain cortex vessels during the fetal period. Med Sci Monit. 2002;8(3):MT46-51. PMID:11887041

29. Harris TH, Banigan EJ, Christian DA, Konradt C, Tait Wojno ED, Norose $\mathrm{K}$ et al. Generalized Lévy walks and the role of chemokines in migration of effector CD8+ T cells. Nature. 2012;486(7404):545-8. https://doi.org/10.1038/ 
Safaeifard et al.

nature 11098 PMID:22722867

30. Tsallis C, Bukman DJ. Anomalous diffusion in the presence of external forces: exact time-dependent solutions and their thermostatistical basis. Phys Rev E Stat Phys Plasmas Fluids Relat Interdiscip Topics. 1996;54(3):R2197-200. https:// doi.org/10.1103/PhysRevE.54.R2197 PMID:9965440

31. Upadhyaya A, Rieu J-P, Glazier JA, Sawada Y. Anomalous diffusion and non-Gaussian velocity distribution of Hydra cells in cellular aggregates. Phys A Stat Mech its Appl. 2001 15;293(3-4):549-58. https://doi.org/10.1016/ S03784371(01)00009-7.

32. Souza Vilela Podestá T, Venzel Rosembach T, Aparecida Dos Santos A, Lobato Martins M. Anomalous diffusion and q-Weibull velocity distributions in epithelial cell migration. PLoS One. 2017 Jul;12(7):e0180777. https://doi.org/ 10.1371/journal.pone.0180777 PMID:28700652

33. Tang X, Wadsworth WG. SAX-3 (Robo) and UNC-40 (DCC) Regulate a Directional Bias for Axon Guidance in Response to Multiple Extracellular Cues. Labrador J-P, editor. PLoS One. 2014;15;9(10):e110031.

34. Nishikawa M, Hörning M, Ueda M, Shibata T. Excitable signal transduction induces both spontaneous and directional cell asymmetries in the phosphatidylinositol lipid signaling system for eukaryotic chemotaxis. Biophys J. 2014;106(3):723-34. https://doi.org/10.1016/j. bpj.2013.12.023 PMID:24507613

35. Li S, Huang NF, Hsu S. Mechanotransduction in endothelial cell migration. J Cell Biochem. 2005;96(6):1110-26. https://doi.org/10.1002/jcb.20614 PMID:16167340

36. Vermolen FJ, Mul MM, Gefen A. Semi-stochastic cell-level computational modeling of the immune system response to bacterial infections and the effects of antibiotics. Biomech Model Mechanobiol. 2014;13(4):713-34. https://doi. org/ 10.1007/s10237-013-0529-5 PMID:24068598

37. Coelho FM, Natale D, Soriano SF, Hons M, Swoger J, Mayer J et al. Naive B-cell trafficking is shaped by local chemokine availability and LFA-1-independent stromal interactions. Blood. 2013;121(20):4101-9. https://doi. org/10.1182/blood-2012-10-465336 PMID:23558016

38. Vroomans RM, Marée AF, de Boer RJ, Beltman JB. Chemotactic migration of $\mathrm{T}$ cells towards dendritic cells promotes the detection of rare antigens. PLOS Comput Biol. 2012;8(11):e1002763.https://doi.org/10.1371/ journal.pcbi. 1002763 PMID:23166480

39. Yang K, Wu J, Xu G, Xie D, Peretz-Soroka H, Santos S, et al. A dual-docking microfluidic cell migration assay (D2Chip) for testing neutrophil chemotaxis and the memory effect. Integrative Biology. 2017;9(4):303-12. https://dx.doi. org $/ 10.1039 \% 2 \mathrm{Fc} 7 \mathrm{ib} 00037 \mathrm{e}$

40. Liu X, Asokan SB, Bear JE, Haugh JM. Quantitative analysis of B-lymphocyte migration directed by CXCL13. Integr Biol (United Kingdom). 2016;8(8).

41. Abeddoust M, Shamloo A. A model for cell density effect on stress fiber alignment and collective directional migration. Phys Biol. 2015;12(6):066023.https://doi.org/10.1088 /1478-3975/12/6/066023 PMID:26717999
42. Vedula SR, Leong MC, Lai TL, Hersen P, Kabla AJ, Lim $\mathrm{CT}$ et al. Emerging modes of collective cell migration induced by geometrical constraints. Proc Natl Acad Sci USA. 2012;109(32):12974-9._https://doi.org/10.1073 /pnas. 1119313109 PMID:22814373

43. Niculescu I, Textor J, de Boer RJ. Crawling and Gliding: A Computational Model for Shape-Driven Cell Migration. PLOS Comput Biol. 2015;11(10):e1004280. https://doi. org/10.1371/ journal.pcbi.1004280 PMID: 26488304

44. Muzzio NE, Pasquale MA, González PH, Arvia AJ. Influence of individual cell motility on the 2D front roughness dynamics of tumour cell colonies. J Biol Phys. 2014;40(3):285-308. https://doi.org/10.1007/s10867-0149349-9 PMID:24893945

45. Missirlis D, Spatz JP. Combined effects of PEG hydrogel elasticity and cell-adhesive coating on fibroblast adhesion and persistent migration. Biomacromolecules.2014;15(1):195-205.https://doi.org/10.1021/ bm4014827 PMID:24274760

46. Raab M, Swift J, Dingal PC, Shah P, Shin JW, Discher DE. Crawling from soft to stiff matrix polarizes the cytoskeleton and phosphoregulates myosin-II heavy chain. J Cell Biol. 2012;199(4):669-83. https://doi.org/10.1083/jcb. 201205056 PMID:23128239

47. Novikova EA, Raab M, Discher DE, Storm C. Persistence-Driven Durotaxis: Generic, Directed Motility in Rigidity Gradients. Phys Rev Lett. 2017;118(7):078103. https://doi.org/10.1103/PhysRevLett.118.078103 PMID:28256894

48. Reymann AC, Boujemaa-Paterski R, Martiel JL, Guérin C, Cao W, Chin HF et al. Actin network architecture can determine myosin motor activity. Science. 2012;336(6086):1310-4. https://doi.org/10.1126/science. 1221708 PMID:22679097

49. Chia PH, Chen B, Li P, Rosen MK, Shen K. Local F-actin network links synapse formation and axon branching. Cell. 2014;156(1-2):208-20. https://doi.org/10.1016/j.cell.2013. 12.009 PMID:24439377

50. Colin A, Bonnemay L, Gayrard C, Gautier J, Gueroui Z. Triggering signaling pathways using F-actin self-organization. Sci Rep. 2016;6(1):34657. https://doi.org/10.1038/ srep34657 PMID:27698406

51. Rangarajan R, Zaman MH. Modeling cell migration in 3D: status and challenges. Vol. 2. Cell Adhes Migr. 2008;2(2):106-9. https://doi.org/10.4161/cam.2.2.6211.

52. Li L, Nørrelykke SF, Cox EC. Persistent cell motion in the absence of external signals: a search strategy for eukaryotic cells. PLoS One. 2008;3(5):e2093. https://doi.org/10.1371 journal.pone.0002093 PMID:18461173

53. Metzler R, Jeon JH, Cherstvy AG, Barkai E. Anomalous diffusion models and their properties: non-stationarity, non-ergodicity, and ageing at the centenary of single particle tracking. Phys Chem Chem Phys. 2014;16(44):2412864. https://doi.org/10.1039/ C4CP03465A PMID:25297814

54. Rosser G, Fletcher AG, Maini PK, Baker RE. The effect of sampling rate on observed statistics in a correlated random 
walk. J R Soc Interface. 2013;10(85):20130273. https://doi. org/10.1098/rsif.2013.0273 PMID:23740484

55. Caballero D, Voituriez R, Riveline D. Protrusion fluctuations direct cell motion. Biophys J. 2014 Jul;107(1):34-42. https://doi.org/10.1016/j.bpj.2014.05.002 PMID:24988339

56. Cooper RM, Wingreen NS, Cox EC. An excitable cortex and memory model successfully predicts new pseudopod dynamics. PLoS One. 2012;7(3):e33528. https://doi. org/10.1371/journal.pone.0033528 PMID:22457772

57. Nishimura SI, Ueda M, Sasai M. Non-Brownian dynamics and strategy of amoeboid cell locomotion. Phys Rev E Stat Nonlinear. Soft Matter Phys. 2012;85(4):41909.

58. Ridley AJ, Schwartz MA, Burridge K, Firtel RA, Ginsberg $\mathrm{MH}$, Borisy $\mathrm{G}$, et al. Cell Migration: Integrating Signals from Front to Back. Science (80). 2003 Dec 5;302(5651):1704-9.

59. Van Haastert PJ. A model for a correlated random walk based on the ordered extension of pseudopodia. PLOS Comput Biol. 2010;6(8):e1000874. https://doi.org/10.1371 /journal.pcbi. 1000874 PMID:20711349

60. Bourne HR, Weiner O. Cell polarity: A chemical compass. Nat. 2002;2002:4196902.

61. Maiuri P, Rupprecht JF, Wieser S, Ruprecht V, Bénichou $\mathrm{O}$, Carpi $\mathrm{N}$ et al. Actin flows mediate a universal coupling between cell speed and cell persistence. Cell. 2015;161(2):374-86. https://doi.org/10.1016/j. cell.2015.01.056 PMID:25799384

62. Read MN, Bailey J, Timmis J, Chtanova T. Leukocyte Motility Models Assessed through Simulation and Multi-objective Optimization-Based Model Selection. PLOS Comput Biol. 2016;12 (9):e1005082. https://doi.org/10.1371/ journal.pcbi. 1005082 PMID:27589606

63. Miller MJ, Wei SH, Parker I, Cahalan MD. Two-Photon Imaging of Lymphocyte Motility and Antigen Response in Intact Lymph Node. Science (80-).2002 Jun 7;296(5574):1869-73. $\quad$ https://doi.org/10.1126/science. 1070051 .

64. Banigan EJ, Harris TH, Christian DA, Hunter CA, Liu AJ. Heterogeneous CD8 + T Cell Migration in the Lymph Node in the Absence of Inflammation Revealed by Quantitative Migration Analysis. 2015;1-20.

65. O'Connor MJ, Hauser AE, Haberman AM, Kleinstein SH. Activated germinal centre B cells undergo directed migration. Int J Data Min Bioinform. 2011;5(3):321-31. https:// doi.org/ 10.1504/IJDMB.2011.040387 PMID:21805826

66. Fooksman DR, Schwickert TA, Victora GD, Dustin ML, Nussenzweig MC, Skokos D. Development and migration of plasma cells in the mouse lymph node. Immunity. 2010 Jul;33 (1):118-27. https://doi.org/10.1016/ j.immuni.2010.06.015 PMID:20619695

67. Garcia R, Moss F, Nihongi A, Strickler JR, Göller S, Erdmann $U$ et al. Optimal foraging by zooplankton within patches: the case of Daphnia. Math Biosci. 2007 Jun;207 (2):165-88. https://doi.org/10.1016/j.mbs.2006.11.014 PMID: 17363010

68. Asano S, Ito S, Takahashi K, Furuya K, Kondo M, Sokabe $\mathrm{M}$ et al. Matrix stiffness regulates migration of human lung fibroblasts. Physiol Rep. 2017 May;5(9):e13281. https:// doi.org/10.14814/phy2.13281 PMID:28507166

69. Rieu JP, Upadhyaya A, Glazier JA, Ouchi NB, Sawada Y. Diffusion and deformations of single hydra cells in cellular aggregates. Biophys J. 2000 Oct; 79 (4):1903-14. https://doi. org/10.1016/S0006-3495(00)76440-X PMID:11023896

70. Huang YL, Tung CK, Zheng A, Kim BJ, Wu M. Interstitial flows promote amoeboid over mesenchymal motility of breast cancer cells revealed by a three dimensional microfluidic model. Integr Biol. 2015 Nov;7(11):1402-11. https://doi.org/10.1039/C5IB00115C PMID:26235230

71. Niculescu I, Textor J, de Boer RJ. Crawling and Gliding: A Computational Model for Shape-Driven Cell Migration. PLOS Comput Biol. 2015 Oct; 11(10): e1004280. https:// doi.org/10.1371/journal.pcbi.1004280 PMID:26488304

72. Fricke GM, Letendre KA, Moses ME, Cannon JL. Persistence and Adaptation in Immunity: T Cells Balance the Extent and Thoroughness of Search. PLOS Comput Biol. 2016 Mar;12(3):e1004818. https://doi.org/10.1371 /journal.pcbi.1004818 PMID:26990103

73. Zhou P, Li B, Liu F, Zhang M, Wang Q, Liu Y et al. The epithelial to mesenchymal transition (EMT) and cancer stem cells: implication for treatment resistance in pancreatic cancer. Mol Cancer. 2017 Feb;16(1):52. https://doi.org/ 10.1186/s12943-017-0624-9 PMID:28245823

74. Brabletz T. EMT and MET in metastasis: where are the cancer stem cells? Cancer Cell. 2012 Dec;22(6):699-701. https://doi.org/10.1016/ j.ccr.2012.11.009 PMID:23238008

75. Box GE, Tiao GC. Bayesian inference in statistical analysis. Wiley; 1992. 588 pp. https://doi.org/10.1002 19781118033197.

76. Metzner C, Mark C, Steinwachs J, Lautscham L, Stadler F, Fabry B. Superstatistical analysis and modelling of heterogeneous random walks. Nat Commun. 2015 Jun; 6(1): 7516. https://doi.org/ 10.1038/ncomms8516 PMID:26108639

77. Jones PJ, Sim A, Taylor HB, Bugeon L, Dallman MJ, Pereira $\mathrm{B}$ et al. Inference of random walk models to describe leukocyte migration. Phys Biol. 2015 Sep;12(6):066001. https:// doi.org/10.1088/1478-3975/12/6/066001 PMID:26403334

78. Wu PH, Giri A, Sun SX, Wirtz D. Three-dimensional cell migration does not follow a random walk. Proc Natl Acad Sci USA. 2014 Mar;111(11):3949-54. https://doi.org/ 10.1073 /pnas. 1318967111 PMID:24594603

79. Davis B. Reinforced random walk. Probability Theory and Related Fields. 1990;84(2):203-29.

80. Zhang M, Zhu C. Global existence of solutions to a hyperbolic-parabolic system. Proceedings of the American Mathematical Society. 2007;135(4):1017-27.

81. Levine HA, Sleeman BD, Nilsen-Hamilton M. Mathematical modeling of the onset of capillary formation initiating angiogenesis. J Math Biol. 2001 Mar;42(3):195-238. https://doi.org/10.1007/s002850000037 PMID:11315313

82. Plank MJ, Sleeman BD, Jones PF. A Mathematical Model of an In Vitro Experiment to Investigate Endothelial Cell Migration. J Theor Med. 2002;4(4):251-70. https://doi.org/ $\underline{10.1080 / 10273660310001594200}$. 
83. Jain HV, Jackson TL. A hybrid model of the role of VEGF binding in endothelial cell migration and capillary formation. Front Oncol. 2013 May;3(May):102. PMID:23675570

84. Bibbona E, Panfilo G, Tavella P. The Ornstein-Uhlenbeck process as a model of a low pass filtered white noise. Metrologia. 2008 Dec;45(6):S117-26. https://doi.org/ $\underline{10.1088 / 0026-1394 / 45 / 6 / \text { S17 }}$

85. Uhlenbeck GE, Ornstein LS. On the Theory of the Brownian Motion. Phys Rev. 1930 Sep;36(5):823-41. https://doi. org/ 10.1103/PhysRev.36.823.

86. Dieterich P, Klages R, Preuss R, Schwab A. Anomalous dynamics of cell migration. Proc Natl Acad Sci U S A. 2008;105(2):459-63. https://doi.org/10.1073/ pnas.0707603105. PMC2206558.

87. Stokes CL, Lauffenburger DA, Williams SK. Migration of individual microvessel endothelial cells: stochastic model and parameter measurement. J Cell Sci. 1991;99(Pt 2):41930. PMID: 1885678

88. Snyder S, DeJulius C, Willits RK. Electrical Stimulation Increases Random Migration of Human Dermal Fibro- blasts. Ann Biomed Eng. 2017;45(9):2049-60. https://doi. org/ 10.1007/s10439-017-1849-x PMID:28488217

89. Dunn GA, Brown AF. A unified approach to analysing cell motility. J Cell Sci Suppl. 1987;8 Supplement 8:81102. https://doi.org/10.1242/jcs.1987.Supplement 8.5 PMID:3503898

90. Li L, Cox EC, Flyvbjerg H. 'Dicty dynamics': Dictyostelium motility as persistent random motion. Phys Biol. 2011;8(4):046006. https://doi.org/10.1088/1478-3975/8/4 /046006 PMID:21610290

91. Fürth R. Reinhold. Die Brownsche Bewegung bei Berücksichtigung einer Persistenz der Bewegungsrichtung. Mit Anwendungen auf die Bewegung lebender Infusorien. Z Phys. 1920 ;2(3):244-56.https://doi.org/10.1007/ BF01328731.

92. Selmeczi D, Mosler S, Hagedorn PH, Larsen NB, Flyvbjerg H. Cell motility as persistent random motion: theories from experiments. Biophys J. 2005;89(2):912-31.https://doi. org/10.1529/biophysj.105.061150 PMID:15951372 